\title{
Meta-analysis of age at onset in spastin-associated hereditary spastic paraplegia provides no evidence for a correlation with mutational class
}

\author{
A G Yip, A Dürr, D A Marchuk, A Ashley-Koch, A Hentati, D C Rubinsztein, E Reid
}

J Med Genet 2003;40:e106 (http://www.jmedgenet.com/cgi/content/full/40/9/e106)

$\mathrm{T}$ he hereditary spastic paraplegias (HSPs) are a group of single gene disorders in which the corticospinal tracts fail to develop normally, or degenerate after initially normal development. The HSPs all share the principal clinical feature of progressive lower limb spastic paralysis, and are subdivided into pure and complicated forms, depending on the presence of additional neurological or non-neurological features. ${ }^{12}$

The pure HSPs tend to be associated with neurodegeneration, rather than abnormal development, and histopathological studies in pure HSP show a length-dependent "dying back" of the terminal ends of the corticospinal tract axons, with the longest axons being involved first. ${ }^{1}$ The SPG4 gene, spastin, is the most important pure HSP gene, being responsible for approximately $40 \%$ of definite autosomal dominant pure HSP and a smaller proportion of sporadic cases and cases with uncertain family history. ${ }^{3}{ }^{4}$ The 616 amino acid spastin protein is a widely expressed AAA (ATPases associated with diverse cellular activities) protein. ${ }^{5}$ More than 100 different spastin mutations have been described, including numerous missense, nonsense, frameshift, and splice site mutations, as well as less frequent whole exon deletions. With only a few possible exceptions, the missense mutations are located in the AAA cassette, from amino acids 342-599. Splice site mutations almost exclusively involve exons 5-16. Nonsense and frameshift mutations are scattered across the gene, with the smallest predicted protein consisting of fewer than 40 amino acids, the largest 562 amino acids. ${ }^{2}$

It is likely that the molecular pathological mechanism of truncating and splice site spastin mutations is loss of function. The associated abnormal transcripts may be unstable, and recent data show that mutant spastin protein is absent in fibroblasts from patients with nonsense and frameshift spastin mutations. ${ }^{67}$ These classes of spastin mutation are probably associated with haploinsufficiency, with disease occurring once functioning spastin levels fall below a critical threshold level. Tolerance for reduced dosage of functioning spastin may be very low, as some "leaky" (that is, creating both wild-type and aberrant splice variants) splice site mutations result in only slight reductions in wild-type mRNA expression. ${ }^{8}$ On the other hand, spastin missense mutations may act via a different mechanism. It has been suggested that spastin has a microtubule severing function and that spastin missense mutants bind constitutively to microtubules, perhaps acting in a dominant negative fashion to block the normal function of spastin or unidentified spastin-related proteins. ${ }^{9}$

One approach to resolve the issue of whether spastin missense mutations have a different molecular pathological mechanism to other mutational types is to examine whether mutational class is correlated with clinical features. The main quantifiable clinical variable in autosomal dominant pure HSP families is age at onset of symptoms, and this shows

\section{Key points}

- The hereditary spastic paraplegias (HSPs) are a group of single gene disorders in which the corticospinal tracts fail to develop normally, or degenerate after initial normal development.

- Mutations in the SPG4 gene, spastin, are responsible for circa $40 \%$ of definite autosomal dominant pure HSP.

- The range of spastin mutational classes found in HSP families is very broad. It is likely that nonsense, frameshift and splice site mutations of spastin act via a loss of function mechanism. However, it has been suggested that spastin missense mutations may act via a dominant negative mechanism.

- One approach to resolve the issue of whether spastin missense mutations have a different molecular pathological mechanism to other mutational types is to examine whether mutational class is correlated with clinical features. We therefore carried out a metaanalysis of age at onset $v$ mutational class correlations in HSP caused by spastin mutations, in order to test the null hypothesis that there is no difference in age at onset between groups of families with different classes of spastin mutation.

- Data gathered on 75 families revealed no significant difference in age at onset between HSP patients with missense $v$ other spastin mutational classes, providing no evidence for a genotype-phenotype correlation.

striking variability within and between families with spastin mutations. If all spastin mutational classes act via the same molecular mechanism, then no difference in age at onset would be expected when families are compared by mutational class. On the other hand, if missense mutations act via a dominant negative mechanism and other mutational types act via a loss of function mechanism, then these different pathological processes might be reflected clinically in discernible differences in age at onset. Such a correlation has not previously been identified. ${ }^{3}$ We therefore carried out a meta-analysis of age at onset $v$ mutational class correlations in HSP caused by spastin mutations, in order to test the null hypothesis that there is no difference in age at onset between groups of families with different classes of spastin mutation.

We analysed all studies $(n=4)$ reporting five or more HSP families with spastin mutations that were published before December 2001..$^{31011}$ We obtained age at onset of symptoms

Abbreviations: HSP, hereditary spastic paraplegia 
data for individual affected patients directly from the relevant papers, or from unpublished data. Asymptomatic individuals were excluded from the analysis.

Within each study, we compared age at onset across different mutational classes and then estimated, as the summary "effect", a hazard ratio of non-missense relative to missense mutation families using conditional Cox regression. ${ }^{12}$ We then pooled the resultant hazard ratios and variances using standard methods of meta-analysis as implemented in Stata 7.0 (Stata Corp., TX, USA)..$^{13}{ }^{14}$

In total, we obtained data on 75 families with known spastin mutations. Age at onset data were available from 356 subjects from these families (details of ages at onset and mutations are available on request). The mutations present were divided into four broad groups; 18 of the families had missense mutations, 23 had presumed splice site mutations, 30 had mutations that resulted in premature truncation (either frameshift or nonsense mutations), and 4 families had mutations that were predicted to cause both a splice mutation and a frameshift. Table 1 shows the numbers of patients with each class of mutation, unweighted mean age at onset and the mean age at onset weighted by family membership. Individual study and pooled hazard ratios for missense mutations relative to all other classes of mutations are shown in table 2. The hazard ratio was homogeneous across studies and there was no significant difference in age at onset in families with missense mutations $v$ families with other classes of mutation.

The pooled data from 75 families confirmed several previously reported observations. The mutational spectrum associated with spastin is wide, and most mutations involve truncation of the molecule or abnormal splicing. Nearly all missense mutations are located within the AAA cassette. Although the age at onset of spastin associated HSP is variable, the mean age at onset is in the late third decade of life.

We looked to see whether any clinical difference in age at onset was apparent between HSP patients with missense $v$ other spastin mutational classes. Although patients with missense mutations had a slightly younger mean age at onset (tables 1 and 2), the standard deviations overlapped widely and this difference was not statistically significant. We cannot therefore reject the null hypothesis that there is no correlation between age at onset and mutational class. On the other hand, our data do not exclude the possibility that different mutational classes may act by different molecular pathological mechanisms, as firstly, our sample size may not have been large enough to detect a small difference in age at onset between groups, and secondly, it is feasible that similar ages at onset may arise from different molecular pathological mechanisms. It is likely that direct experimental work or

Table 1 Unweighted and weighted mean age at onset data for each mutational class, for families described in the four studies included in the meta-analysis

\begin{tabular}{|c|c|c|c|c|}
\hline \multirow[b]{2}{*}{ Mutational class } & \multirow[b]{2}{*}{$\begin{array}{l}\text { No of } \\
\text { families }\end{array}$} & \multirow[b]{2}{*}{$\begin{array}{l}\text { No of } \\
\text { subjects }\end{array}$} & \multicolumn{2}{|c|}{ Mean age at onset } \\
\hline & & & $\begin{array}{l}\text { Unweighted } \\
\text { years (SD) } \\
\text { Range (years) }\end{array}$ & $\begin{array}{l}\text { Weighted } \\
\text { by family } \\
\text { years (SD) }\end{array}$ \\
\hline $\begin{array}{l}\text { Frameshift+ } \\
\text { presumed exon } \\
\text { skip }\end{array}$ & 4 & 25 & $\begin{array}{l}28.91(4.98) \\
(<1-62)\end{array}$ & $28.44(5.02)$ \\
\hline Missense & 18 & 69 & $\begin{array}{l}24.88(13.96) \\
(<1-60)\end{array}$ & $25.26(11.31)$ \\
\hline Splice site & 23 & 115 & $\begin{array}{l}29.02(9.63) \\
(<1-60)\end{array}$ & $29.56(8.12)$ \\
\hline Truncating & 30 & 147 & $\begin{array}{l}29.98(10.63) \\
(<1-74)\end{array}$ & $30.37(10.63)$ \\
\hline
\end{tabular}

Table 2 Individual study and pooled hazard ratios for missense mutations relative to all other classes of mutations

\begin{tabular}{lll}
\hline Study & Hazard ratio & $\begin{array}{l}95 \% \text { confidence } \\
\text { interval }\end{array}$ \\
\hline Fontneckten $^{3}$ & 1.33 & 0.86 to 2.04 \\
Hentati $^{11}$ & 0.99 & 0.60 to 1.64 \\
Lindsey $^{10}$ & 1.19 & 0.29 to 4.96 \\
Svenson $^{8}$ & 1.11 & 0.66 to 1.77 \\
Pooled $^{8}$ & 1.14 & 0.78 to 1.50 \\
\hline
\end{tabular}

Test for heterogeneity: $Q=0.79, p=0.85$.

further meta-analyses with very large sample sizes will be required to resolve this issue.

\section{ACKNOWLEDGEMENTS}

We thank the families who took part in the studies on which this analysis was based. DCR is a Wellcome Senior Research Fellow in Clinical Science.

\section{Authors' affiliations}

A G Yip, Genetics Program (Department of Medicine), Boston University School of Medicine, Boston, MA 02118, USA

A Dürr, INSERM U 289 and Département de Génétique, Cytogénétique et Embryologie, Hôpital de la Salpêtrière, Paris, France

D A Marchuk, Department of Molecular Genetics and Microbiology, Duke University Medical Center, Durham, NC 27710, USA

A Ashley-Koch, Center for Human Genetics, Duke University Medical Center, Durham, NC 27710, USA

A Hentati, Department of Neurology, Northwestern University Medical School Chicago, 300 E. Superior Street, Tarry 13-715, Chicago, IL 60611, USA

D C Rubinsztein, E Reid, Department of Medical Genetics, Cambridge Institute for Medical Research, Wellcome Trust/MRC Building, University of Cambridge, Cambridge, UK

Correspondence to: Dr E Reid, Department of Medical Genetics, University of Cambridge, Box 134, Addenbrooke's Hospital, Cambridge CB2 2QQ UK; ereid@hgmp.mrc.ac.uk

\section{REFERENCES}

1 Harding AE. The hereditary ataxias and related disorders. Edinburgh: Churchill Livingstone, 1984

2 Reid E. The hereditary spastic paraplegias. J Neurol 1999;246:995-1003.

3 Fonknechten N, Mavel D, Byrne P, et al. Spectrum of SPG4 mutations in autosomal dominant spastic paraplegia. Hum Mol Genet 2000;9:637-44.

4 Sauter S, Miterski B, Klimpe S, et al. Mutation analysis of the spastin gene (SPG4) in patients in Germany with autosomal dominant hereditary spastic paraplegia. Human Mutation 2003;20:127-32.

5 Hazan J, Fonknechten N, Mavel D, et al. Spastin, a new AAA protein, is altered in the most frequent form of autosomal dominant spastic paraplegia. Nature Genet 1999;23:296-303.

6 Patrono C, Casili C, Tessa A, et al. Missense and splice site mutations in SPG4 suggest loss of function in dominant spastic paraplegia. J Neurol 2002;249:200-5.

7 Charvin D, Cifuentes-Diaz C, Fonknechten N, et al. Mutations of SPG4 are responsible for a loss of function of spastin, an abundant neuronal protein localized in the nucleus. Hum Mol Genet 2003;12(1):71-8.

8 Svenson IK, Ashley-Koch AE, Gaskell PC, et al. Identification and expression analysis of spastin gene mutations in hereditary spastic paraplegia. Am J Hum Genet 2001:68:1077-85

9 Errico A, Ballabio A, Rugarli El. Spastin, the protein mutated in autosomal dominant hereditary spastic paraplegia, is involved in microtubule dynamics. Hum Mol Genet 2002;11:153-63.

10 Lindsey JC, Lusher ME, McDermott CJ, et al. Mutation analysis of the spastin gene (SPG4) in patients with hereditary spastic paraparesis. J Med Genet 2000;37:759-65.

11 Hentati A, Deng HX, Zhai $\mathrm{H}$, et al. Novel mutations in spastin gene and absence of correlation with age at onset of symptoms. Neurology 2000;55(9): 1388-90.

12 Cox DR, Oakes D. Analysis of survival data. London: Chapman and Hall, 1984.

13 Sutton AJ, Abrams KR, Jones DR. Meta-analysis of survival data. In: Systematic reviews of trials and other studies. Health Technol Assess 1998;2(19): 169-75.

14 Sharp S, Sterne J. SBE16: Meta-analysis. Stata Tech Bull 1997;38:9-14. 